Research Paper

\title{
Circulating Lymphocytes, PD-L1 Expression on Tumor-infiltrating Lymphocytes, and Survival of Colorectal Cancer Patients with Different Mismatch Repair Gene Status
}

Pengfei Kong1, 2*, Jing Wang ${ }^{1,3^{*}}$, Ze Song1,4, Shousheng Liu1 ${ }^{1,2}$, Wenzhuo He ${ }^{1,2}$, Chang Jiang1,2 Qianku $\mathrm{Xie}^{1,2}$, Lin Yang ${ }^{1,2}$, Xiaojun Xia ${ }^{\circledR}$, Liangping $\mathrm{Xia}^{1,2}{ }^{\circledR}$

1. State Key Laboratory of Oncology in South China, Collaborative Innovation Centre for Cancer Medicine, Sun Yat-sen University Cancer Center, Guangzhou, 510060, PR China;

2. Department of the VIP region, Sun Yat-sen University Cancer Center, Guangzhou, 510060, PR China;

3. Department of Molecular Diagnostics, Sun Yat-sen University Cancer Center, Guangzhou, 510060, PR China;

4. Department of the Medical Imaging and Interventional Radiology, Sun Yat-sen University Cancer Centre, Guangzhou, 510000, PR China.

* Equal contributors.

$\triangle$ Corresponding authors: Xiaojun Xia, PhD; E-mail: xiaxj@sysucc.org.cn and Liangping Xia, MD, PhD; E-mail: xialp@sysucc.org.cn

(C) Ivyspring International Publisher. This is an open access article distributed under the terms of the Creative Commons Attribution (CC BY-NC) license (https://creativecommons.org/licenses/by-nc/4.0/). See http://ivyspring.com/terms for full terms and conditions.

Received: 2018.10.03; Accepted: 2019.01.02; Published: 2019.04.05

\begin{abstract}
Clinical outcomes of checkpoint blockade immunotherapy on colorectal cancer (CRC) are influenced by mismatch repair (MMR) gene status, which is associated with distinct tumor immune infiltrates and systemic inflammatory response status. However, the prognostic value of PD-L1 expression and the systemic inflammatory response for patients with MMR deficiency has not been fully investigated. In this study, we examined the association of systemic inflammatory markers, PD-1/PD-LI pathway expression, microsatellite instability (MSI) status, and clinicopathological characteristics of CRC with patient survival between MMR-deficient (dMMR) group $(\mathrm{N}=168)$ and MMR-proficient (pMMR) group $(\mathrm{N}=169)$. We found a large proportion of dMMR CRC patients displayed increased level of systemic inflammatory markers such as C-reactive protein, Neutrophil/Lymphocyte Ratio (NLR), Glasgow Prognostic Score (GPS), and low expression of PD-L1 in tumor stroma. Several systemic inflammatory markers were associated with AJCC stage only in dMMR patients. Similarly, Tumor infiltrating lymphocyte (TIL) PD-L1 or stroma PD-L1 expression was associated with AJCC stage only in dMMR patients. Circulating serum lymphocytes and TIL PD-LI expression are both independent prognosis predictors for CRC patients. Overall, we found that $\mathrm{DMMR}$ CRC displayed a comprehensively distinct tumor immune microenvironment and systemic inflammatory response makers. PD-LI expression at different location has different impacts on CRC patient survival, and the TIL PD-LI expression might be a potential predictor for dMMR CRC patient response to anti-PD-1 therapy.
\end{abstract}

Key words: PD-L1, PD-1, tumor infiltrating lymphocytes, colorectal cancer, immunotherapy, predictor

\section{Background}

Cancer is a heterogeneous disease with multiple genetic events and subsequent clonal evolution, which potentially generate numerous tumor neoantigens monitored by the immune system, which activate antitumor immune response to eradicate or control tumor growth, which is the essence of cancer immunosurveillance [1-3]. On the other hand, tumors also develop multiple strategies to avoid immune attack and even actively suppress immune system. One aspect of the immunoediting hypothesis is that 
tumors can evade immune surveillance by expressing molecules inhibiting the anti-tumor immune response, including immuno-suppressive cytokines such as IL-10 (Interleukin-10) [4], transforming growth factor$\beta$ (TGF- $\beta$ )[5], and immune co-inhibitory signaling proteins such as Programmed cell death protein 1 (PD-1), programmed cell death 1 ligand 1 (PD-L1) and cytotoxic T lymphocyte-associated antigen 4 (CTLA4) [6], etc. The discovery of the co-inhibitory pathways boost the antibody blockade therapeutics targeting these molecules, such as anti-PD-1, anti-PD-L1 and anti-CTLA4 [7, 8]. Up to now, these therapies have achieved noteworthy efficacy in multiple types of cancers by blocking cancer immune evasion and enabling patients to reinvigorate an effective anti-cancer response[8-13]. Nevertheless, the efficacy of checkpoint blockade antibodies was far from satisfying in CRC patients [13, 14].

Previously, tumors with a high burden of neoantigens and low intratumor heterogeneity were found to be significantly more responding to immunotherapy [15]. Recently, several studies reported that mismatch repair (MMR) gene MSH2, MSH6,MLH1 and PMS2 are involved in DNA repair and loss of the proteins can result in frameshift mutations and potentially cause high neoantigen burden[1, 16, 17]. Accordingly, tumors with MMR-deficiency (dMMR) may have a relatively high neoantigen spectrum, which subsequently induce $\mathrm{T}$ lymphocytes infiltration and anti-tumor immune response, and form totally different tumor immune microenvironment (TME) from that of MMR-proficient (pMMR) tumors. A recent clinical trial suggested that only the CRC patients with $\mathrm{dMMR}$ derived clinical benefit from anti-PD-1 therapy[14]. The rationale is that dMMR CRC has higher mutation rate and potentially generate more neoantigens, which serve as targets for anti-tumor immunity recovered by PD-1 blockade. Nevertheless, the exact mechanisms underlying such superior efficacy in this specific subtype of CRC patients are still not completely clear. Furthermore, the prognostic value of PD-1/PD-L1 expression and the systemic inflammatory response markers for dMMR CRC patients has not been fully investigated. Therefore, we designed a study to identify the difference of systemic and local inflammation-related, PD-1/PD-L1 pathway-related TME between dMMR and pMMR CRC, and to explore potential prognosis prediction markers for CRC immunotherapy.

\section{Materials and Methods}

\section{Study design and participants}

A single center study was conducted at the Sun Yat-sen University Cancer Center (SYSUCC), and the database of our institution contains more than 4000 CRC patients who were admitted between January 2003 and October 2015. This study was approved by SYSUCC institutional review board, and all the participants signed informed consent forms. Among these patients, a total of 2,901 CRC patients underwent MSI testing from May 2011 to Jane 2015, and 244 $(8.4 \%)$ were with dMMR status. In addition, we excluded patients with following criteria: cancer other than CRC, stage unknown, and vital status unknown or lost to follow-up. Next, we identified another 169 patients of pMMR status matched with the dMMR patients in clinicopathological characteristics (Figure 1). Characteristics of pMMR patients enrolled and total were compared using multivariable analysis (Table S1).

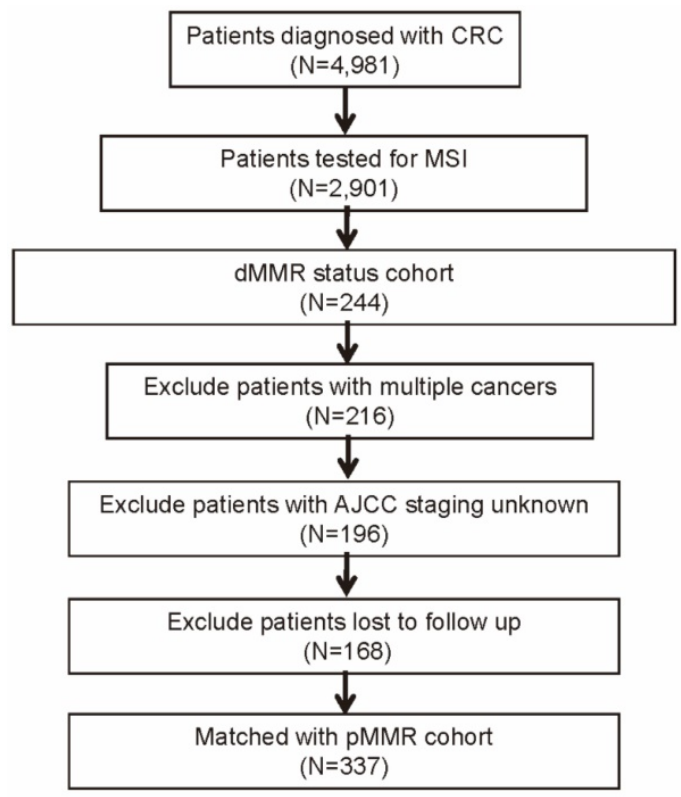

Figure 1. Flow diagram of patient selection procedure.

All enrolled patients were confirmed the diagnosis of CRC and the pathological type by pre-therapy pathologic examination. Age, sex, tumor location, tumor grade, histological subtype, tumor stage, regional lymph nodes (LNs) metastasis, distant metastasis, American Joint Committee on Cancer (AJCC) 7th edition staging, perineural invasion, vascular invasion, number of retrieved LNs, chemotherapy status, radiotherapy status, serum inflammatory factors, and serum tumor markers, such as carcinoembryonic antigen (CEA) (upper physiological value: $5 \mathrm{ng} / \mathrm{ml}$ ) and carbohydrate antigen 19-9 (CA19-9) (upper physiological value: $30 \mathrm{U} / \mathrm{ml}$ ) were retrospectively reviewed. Follow-up examinations including computed tomography (CT) scans were conducted regularly. Furthermore, we routinely obtained the follow-up data from the institutional 
database, which was constantly updated based on physician records, tumor registry information, and telephone interviews.

\section{Immunohistochemical analysis of MMR proteins, PD-1, and PD-L1 expression}

For immunohistochemical (IHC) staining of markers in the tumor tissues, $4 \mu \mathrm{m}$-thick unstained histological sections from each formalin-fixed, paraffin-embedded tissue block were de-waxed with xylene and rehydrated in $100 \%, 95 \%$, and $70 \%$ ethanol to water. Analysis for MMR-related proteins was conducted as previously described [18]. For antigen retrieval, the slides were dipped in EDTA $(\mathrm{PH}=8.0)$ and heated in an autoclave for 10 minutes. Endogenous peroxidase activity was quenched through incubation with $3 \% \mathrm{H}_{2} \mathrm{O}_{2}$ for 10 minutes. Slides were incubated at $37^{\circ} \mathrm{C}$ for 50 minutes with diluted monoclonal antibodies (dilution 1:50) as follows: anti-hMLH1 (ZSGB-BIO Corp, ZM-0154, Beijing, China), anti-hMSH2 (ZSGB-BIO Corp, ZA-0622, Beijing, China), anti-hMSH6 (ZSGB-BIO Corp, ZA-0541, Beijing, China), and anti-hPMS2 (ZSGB-BIO Corp, ZA-0542, Beijing, China) antibodies. Slides were then processed using a DAB Detection kit (ZSGB-BIO Corp, PV-6000-D, Beijing, China) according to the manufacturer's instructions. Finally, the sections were incubated with diaminobenzidine, counterstained with haematoxylin, dehydrated in ascending graded of ethanol and mounted. In accordance with previous studies, the immunostaining was classified into two grades for MMR proteins $(-, \leq 10 \%$; ,$+>10 \%$ nuclear staining) (Figure S1) $[19,20]$.

IHC staining for PD-1 and PD-L1 expression was performed as described in previous study [21, 22]. The primary antibodies were used as follows: anti-PD-1 (CST Corp, \#10084, Massachusetts, USA), anti-PD-L1 (CST Corp, \#10084, Massachusetts, USA), and DAKO Envision kit (DAKO Corp, Carpinteria, CA, USA). In addition, PD-1 and PD-L1 immunostaining was divided into two groups based on intensity and extent $(-, \leq 5 \% ;+,>5 \%$, membranous staining) $(0, \leq 5 \% ; 1,6 \%$ to $25 \% ; 2,26 \%$ to $50 \% ; 3,51 \%$ to $75 \% ; 4,>75 \%$ ) (Figure S2).[23]

\section{Tumor infiltrating lymphocytes evaluation}

Tumor-infiltrating lymphocytes (TILs) was assessed in HE-stained sections as previously reported [24]. Only small blue mononuclear cells that typically had a halo around tumor cells were counted. Samples were analyzed according to the mean TIL/high-powered field (HPF) calculated for each tumor. The cutoff value of mean TIL number/HPF was determined using receiver operating characteristic (ROC) curve analyses. Tumors were then divided into two groups (TIL/HPF $\geq 2$ or $\mathrm{TIL} / \mathrm{HPF}<2$ ) according to the cutoff value. The expression of PD1 and PD-L1 in TILs was estimated using IHC method mentioned above.

\section{Definition of inflammation-based prognostic score}

Based on a previous study, the Glasgow Prognostic Score (GPS) was calculated as follows: patients with elevated levels of both C-reactive protein (CRP) (>10 mg/dl) and hypo-albuminemia $(<3.5 \mathrm{~g} / \mathrm{dl})$ were allocated a score of 2 , and patients showing only one or none of these blood chemistry abnormalities were allocated a score of 1 or 0 , respectively. The NLR was calculated as: NLR= neutrophil ratio (\%) (or number of neutrophils)/ Lymphocyte ratio (\%) (or number of lymphocytes). The CAR was calculated as: $\mathrm{CAR}=$ serum $\mathrm{CRP}$ level $(\mathrm{mg} / \mathrm{dl}) /$ serum albumin level (g/dl)[25].

\section{Statistical analysis}

All the statistical analysis was conducted with SPSS software (Version 19.0, SPSS). Patients' clinicopathological characteristics and the associations between PD-L1and MMR markers expression were evaluated using the Fisher's exact test or the $\chi^{2}$ tests. The primary endpoints - overall survival (OS) and disease-free survival (DFS) were calculated using the Kaplan-Meier method, and survival curves were compared using the log-rank test method. The Cox-regression analysis, both univariate and multivariate, was performed to identify the independency of prognostic factors status. Hazard ratios (HR) and 95\% confidence intervals (CIs) were used to determine the prognostic impact of factors on survival time for univariate models, or multivariate models adjusted for variants including MMR status, age, gender, tumor location, tumor grade, histological subtype, LNs metastasis, distant metastasis, perineural invasion, vascular invasion, number of retrieved LNs, therapy status (chemotherapy and radiotherapy), and AJCC staging. All tests were two-sided, and a $P$ value $<0.05$ was considered to be statistically significant.

\section{Results}

\section{Baseline patient characteristics}

The patient selection is presented in Figure $\mathbf{1}$ (and detailed in the Methods section). Comparison of clinicopathological characteristics between dMMR and pMMR CRC patients was listed in Table 1. Of the 337 CRC patients, 169 (50.1\%) of the patients were pMMR and 168(49.9\%) were dMMR. Patients with dMMR presented at a younger age ( $53.5 \mathrm{v} 58.5$ years, $P<0.001$ ), and were more frequently found on right hemicolon for tumor location $(46.4 \% \mathrm{v} 27.8 \%, P=0.001)$ 
and grade $3(46.4 \%$ v $26.0 \%, P<0.001)$ for tumor grade, and more with mucinous histological subtype ( $35.7 \%$ $\mathrm{v} 17.8 \%, P<0.001)$. In addition, a significant lower proportion of $\mathrm{dMMR}$ patients were with advanced tumor stage. There was no significant difference between the two groups in gender, regional LNs metastasis, distant metastasis, AJCC stage, perineural invasion, vascular invasion, number of retrieved LNs, chemotherapy, and radiotherapy treatment.

Table 1. Comparison of clinicopathological characteristics between $\mathrm{dMMR}$ and PMMR CRC patients.

\begin{tabular}{|c|c|c|c|c|c|}
\hline \multirow[b]{2}{*}{ Variable } & \multicolumn{2}{|l|}{ dMMR } & \multicolumn{2}{|l|}{ pMMR } & \multirow[b]{2}{*}{$P$} \\
\hline & $\begin{array}{l}\text { No. of Patients } \\
(\mathrm{n}=168)\end{array}$ & $\%$ & $\begin{array}{l}\text { No. of Patients } \\
(n=169)\end{array}$ & $\%$ & \\
\hline \multicolumn{5}{|l|}{ Age (years) } & $<0.001$ \\
\hline Mean & 53.5 & & 58.5 & & \\
\hline SD & 12.5 & & 11.7 & & \\
\hline \multicolumn{5}{|l|}{ Gender } & 0.219 \\
\hline Male & 109 & 64.9 & 98 & 58.0 & \\
\hline Female & 59 & 35.1 & 71 & 42.0 & \\
\hline \multicolumn{5}{|c|}{ Tumor location } & 0.001 \\
\hline Right hemicolon & 78 & 46.4 & 47 & 27.8 & \\
\hline Left hemicolon & 54 & 32.1 & 83 & 49.1 & \\
\hline Rectum & 36 & 21.4 & 39 & 23.1 & \\
\hline \multicolumn{5}{|l|}{ Tumor grade } & $<0.001$ \\
\hline 1 & 1 & 0.6 & 2 & 1.2 & \\
\hline 2 & 89 & 53.0 & 123 & 72.8 & \\
\hline 3 & 78 & 46.4 & 44 & 26.0 & \\
\hline \multicolumn{5}{|c|}{ Histological subtype } & $<0.001$ \\
\hline Mucinous & 60 & 35.7 & 30 & 17.8 & \\
\hline Other & 108 & 64.3 & 139 & 82.2 & \\
\hline \multicolumn{5}{|l|}{ Tumor stage } & 0.003 \\
\hline $\mathrm{T}_{1}$ & 5 & 3.0 & 8 & 4.7 & \\
\hline $\mathrm{T}_{2}$ & 24 & 14.3 & 25 & 14.8 & \\
\hline $\mathrm{T}_{3}$ & 96 & 57.1 & 120 & 71.0 & \\
\hline $\mathrm{T}_{4 \mathrm{a}}$ & 30 & 17.9 & 11 & 6.5 & \\
\hline $\mathrm{T}_{4 \mathrm{~b}}$ & 13 & 7.7 & 5 & 3.0 & \\
\hline \multicolumn{5}{|c|}{ Regional LNs metastasis } & 0.155 \\
\hline $\mathrm{N}_{0}$ & 122 & 72.6 & 121 & 71.6 & \\
\hline $\mathrm{N}_{1}$ & 37 & 22.0 & 30 & 17.8 & \\
\hline $\mathrm{N}_{2}$ & 9 & 5.4 & 18 & 10.7 & \\
\hline \multicolumn{5}{|c|}{ Distant metastasis } & 0.653 \\
\hline $\mathrm{M}_{0}$ & 157 & 93.5 & 160 & 94.7 & \\
\hline $\mathrm{M}_{1}$ & 11 & 6.5 & 9 & 5.3 & \\
\hline \multicolumn{5}{|l|}{ AJCC stage } & 0.971 \\
\hline 1 & 26 & 15.5 & 27 & 16.0 & \\
\hline 2 & 89 & 53.0 & 91 & 53.8 & \\
\hline 3 & 42 & 25.0 & 42 & 24.9 & \\
\hline 4 & 11 & 6.5 & 9 & 5.3 & \\
\hline \multicolumn{5}{|c|}{ Perineural invasion } & 0.129 \\
\hline Negative & 153 & 91.1 & 144 & 85.2 & \\
\hline Positive & 15 & 8.9 & 25 & 14.8 & \\
\hline \multicolumn{5}{|c|}{ Vascular invasion } & 0.080 \\
\hline Negative & 155 & 92.3 & 145 & 85.8 & \\
\hline Positive & 13 & 7.7 & 24 & 14.2 & \\
\hline \multicolumn{5}{|c|}{ Number of retrieved LNs } & 0.304 \\
\hline$<12$ & 54 & 33.3 & 66 & 39.1 & \\
\hline$\geq 12$ & 108 & 66.7 & 103 & 60.9 & \\
\hline \multicolumn{4}{|l|}{ Chemotherapy } & & 0.304 \\
\hline Yes & 69 & 33.3 & 97 & 39.1 & \\
\hline No & 99 & 66.7 & 72 & 60.9 & \\
\hline \multicolumn{4}{|l|}{ Radiotherapy } & & 0.368 \\
\hline Yes & 12 & 7.1 & 8 & 4.7 & \\
\hline No & 156 & 92.9 & 161 & 95.3 & \\
\hline
\end{tabular}

\section{Serum tumor markers and systemic inflammatory markers}

CRC patients with pMMR were more likely to present a high level of circulating serum CEA $(46.2 \% \mathrm{v}$ $35.1 \%, P=0.046$ ) (Table 2). In addition, there was significant difference between the two groups in systemic inflammatory makers including C-reactive protein $(<8.2 / \geq 8.2)(\mathrm{mg} / \mathrm{L})$, NLR $(<1.8 / \geq 1.8)$, and GPS $(0 / 1 / 2)$.Specifically, a larger proportion of dMMR group showed high level of C-reactive protein $38.7 \%$ v $17.2 \%, P<0.001)$, high level of NLR $(80.4 \%$ v $68.6 \%$, $P=0.017$ ), and GPS (core 1, 30.4\% v 14.2\%, $P<0.001$ ). The circulating lymphocytes level was not associated with MMR status, and showed a decreasing trend in stage 4 CRC patients; By contrast, NLR levels significantly increased in stage 4 patients (Figure 2B-F). Similarly, several other inflammatory factors like CRP, CAR, and GPS, significantly increased in late stage $\mathrm{pMMR}$ group, and exhibited an increasing tendency in late stage dMMR patients (Figure S3).
A
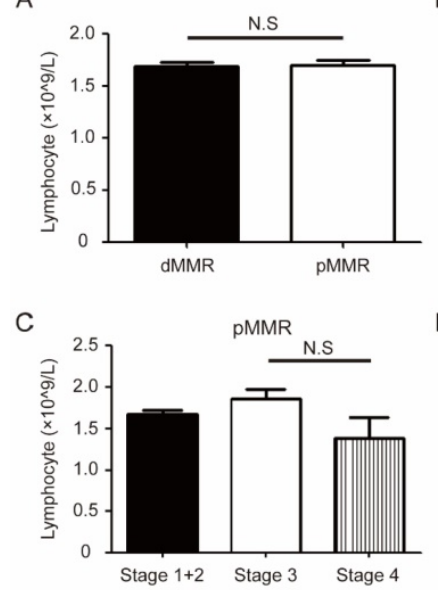

E

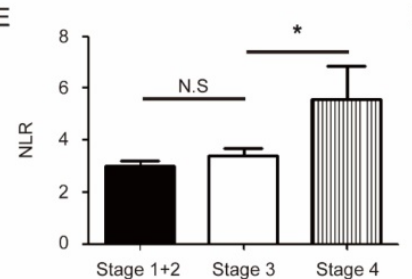

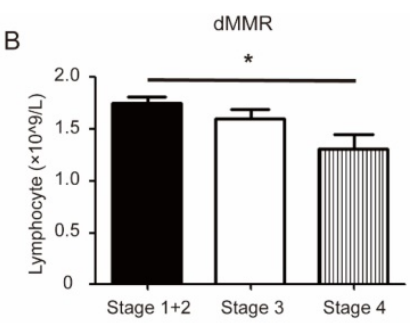

D
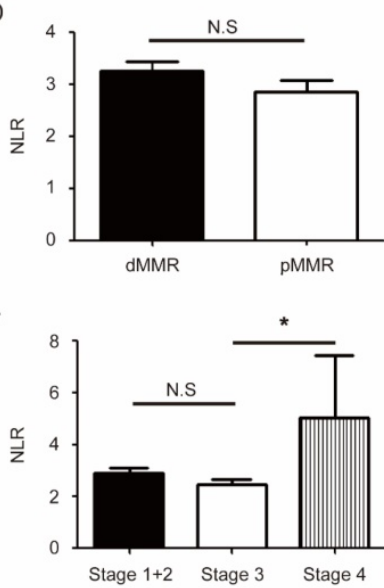

Figure 2. Different inflammatory status between $d M M R$ and $P M M R$ CRC patients. (a). The level of circulating lymphocytes in AMMR and PMMR CRC patients. (b-c). Group comparison combined the level of circulating lymphocytes and AJCC stages in dMMR patients (b) or pMMR patients (c). (d). The level of neutrophil to lymphocyte ratio (NLR) in $\mathrm{DMMR}$ and pMMR CRC patients. (e-f). Group comparison combined the level of NLR and AJCC stages in dMMR patients (e) or pMMR patients (f). $* P<0.05$, N.S, not significant.

\section{PD-1/PD-L1 axis in AMMR and PMMR CRC patients}

All PD-1/PD-L1 pathway results between two cohorts are shown in Figure 3 and Table 3. Total PD-1 
and PD-L1 positive expression rate were 60.5\% (204/337) and 51.9\% (175/337), respectively. When stratified with MMR status, positive staining ratio of total PD-1 (62.5\% v 58.6\%, P=0.504) and PD-L1 (55.4\% v $48.5 \%, P=0.231$ ) was similar between two groups. We analyzed the distribution pattern of PD-1 and PD-L1 staining on different areas (total, tumor, TIL, and stroma) (Figure 3A-B), and the patterns of PD-1 and PD-L1 staining were also very similar in the two CRC groups. Among the various PD-1/PD-L1 expression patterns, dMMR patients showed higher TIL PD-1 (54.8\% v 43.2\%, $P=0.039)$ and lower stroma PD-L1 (non-TIL and non-tumor) expression $(22.0 \% \mathrm{v}$ $43.8 \%, P<0.001)$. Importantly, late stage dMMR patients, but not pMMR patients, showed significantly lower level of TIL PD-L1 or stroma PD-L1 (Figure 3C-F). Besides, MMR status is not associated with PD-1/PD-L1 staining patterns on different areas (Table 3).

\section{Prognostic factors in dMMR and pMMR CRC patients}

Overall survival (OS) and disease-free survival (DFS) curves are displayed in Figure 4. OS and DFS between the dMMR and pMMR groups were not significantly different. Interestingly, low level of circulating lymphocytes $\left(<1.12 \times 10^{9} / \mathrm{L}\right)$, is associated with short OS $(P=0.022)$ and short DFS $(P=0.002)$ only in $\mathrm{dMMR} C \mathrm{CRC}$, but not in pMMR CRC patients (Figure 4A-D). Consistently, circulating lymphocyte levels is lower in dMMR patients with short DFS when stratified with different DFS $(>3$ years and $<3$ years) (Figure 4E -F).

Next, to determine whether the parameters examined above have other prognostic relevance, we conducted univariate and multivariate analysis in our patients (Table 4-5). At the end of follow-up, 13 patients $(7.7 \%)$ died in the dMMR cohorts and 9 patients $(5.3 \%)$ died in the pMMR cohorts. In addition, 27 patients $(16.1 \%)$ relapsed in the dMMR cohorts and 14 patients (8.3\%) relapsed in the pMMR cohorts. OS and DFS between the dMMR and pMMR groups were not significantly different. The multivariate analyses results indicated that factors associated with decreased OS included distant metastasis(multivariable Cox HR, 5.23; 95\% CI, 1.54 to 17.83; $P=0.008$ ), advanced AJCC stage (multivariable Cox HR, 7.35; 95\% CI, 1.01 to 53.69; $P=0.049$ ), vascular invasion(multivariable Cox HR, 4.98; 95\% CI, 1.63 to 15.24; $P=0.005$ ), radiotherapy (multivariable Cox HR, 4.40; 95\% CI, 1.49 to $12.93 ; P=0.006)$, and low lymphocytes level $(<1.12 \times 109 / \mathrm{L})$ (multivariable Cox HR, 4.82; 95\% CI, 1.12 to 20.69; $P=0.035$ ) (Table 4). On the other hand, factors correlated with short DFS include distant metastasis (multivariable Cox HR,
4.39; 95\% CI, 1.58 to $12.21 ; P=0.005)$, number of retrieved LNs $(<12)$ (multivariable Cox HR, 2.01; 95\% $\mathrm{CI}, 1.00$ to $4.03 ; P=0.049)$, and radiotherapy (multivariable Cox HR, 6.80; 95\% CI, 2.86 to 16.17; $P<0.001) \quad$ (Table 5). Multivariate analysis after adjustment identified that the two groups have similar independent prognostic factors for OS and DFS (Table S2-5).

\section{PD-LI expression at different location has different impact on survival of CRC patients}

Our initial results suggested that total PD-1 expression was not a prognostic marker, but the total PD-L1 expression was a good prognostic marker (Figure S4). When stratified with PD-L1 expression pattern, OS is not associated with either tumor PD-L1 expression $(P=0.838)$ (Figure 5A) or stroma PD-L1 expression $(P=0.356)$ (Figure 5B). Strikingly, high TIL PD-L1 expression significantly correlated with long OS and DFS of CRC patients $(P=0.019$ for OS, $P=0.030$ for DFS), while PD-L1 expression in other areas has no association with survival (Figure 5C-F). By contrast, either total or localized PD-1 expression pattern was not associated with survival at all (Figure S5).

Table. 2 Comparison of tumor markers and systemic inflammatory status between $\mathrm{dMMR}$ and $\mathrm{PMMR} C R C$ patients.

\begin{tabular}{|c|c|c|c|c|c|}
\hline \multirow[b]{2}{*}{ Variable } & \multicolumn{2}{|l|}{ dMMR } & \multicolumn{2}{|l|}{ pMMR } & \multirow[b]{2}{*}{$P$} \\
\hline & $\begin{array}{l}\text { No. of Patients } \\
(n=168)\end{array}$ & $\%$ & $\begin{array}{l}\text { No. of Patients } \\
(n=169)\end{array}$ & $\%$ & \\
\hline CEA $(\mu \mathrm{g} / \mathrm{ml})$ & & & & & 0.046 \\
\hline$\geq 5.0$ & 59 & 35.1 & 78 & 46.2 & \\
\hline$<5.0$ & 109 & 64.9 & 91 & 53.8 & \\
\hline \multicolumn{2}{|c|}{ CA19-9 (units/ml) } & & & & 0.790 \\
\hline$\geq 30$ & 36 & 21.4 & 34 & 20.1 & \\
\hline$<30$ & 132 & 78.6 & 135 & 79.9 & \\
\hline \multicolumn{2}{|l|}{ Albumin $(\mathrm{g} / \mathrm{l})$} & & & & 0.464 \\
\hline$\geq 35$ & 154 & 91.7 & 150 & 88.8 & \\
\hline$<35$ & 14 & 8.3 & 19 & 11.2 & \\
\hline \multicolumn{3}{|c|}{ C-reactive protein $(\mathrm{mg} / \mathrm{l})$} & & & $<0.001$ \\
\hline$\geq 8.2$ & 65 & 38.7 & 29 & 17.2 & \\
\hline$<8.2$ & 103 & 61.3 & 140 & 82.8 & \\
\hline \multicolumn{3}{|c|}{ Neutrophil (×109/1) } & & & 0.248 \\
\hline$<2.0$ & 5 & 3.0 & 8 & 5.0 & \\
\hline $2.0-7.0$ & 145 & 86.3 & 141 & 88.7 & \\
\hline$>7.0$ & 18 & 10.7 & 10 & 8.6 & \\
\hline \multicolumn{3}{|c|}{ Lymphocyte $\left(\times 10^{9} / 1\right)$} & & & 1.000 \\
\hline$\geq 1.12$ & 162 & 96.4 & 163 & 96.4 & \\
\hline$<1.12$ & 6 & 3.6 & 6 & 3.6 & \\
\hline \multicolumn{2}{|l|}{ CAR $(\mathrm{mg} / \mathrm{g})$} & & & & 0.056 \\
\hline$\geq 0.03$ & 136 & 81.0 & 123 & 71.9 & \\
\hline$<0.03$ & 32 & 19.0 & 48 & 28.1 & \\
\hline \multicolumn{2}{|l|}{ NLR } & & & & 0.017 \\
\hline$\geq 1.8$ & 135 & 80.4 & 116 & 68.6 & \\
\hline$<1.8$ & 33 & 19.6 & 53 & 31.4 & \\
\hline \multicolumn{2}{|l|}{ GPS } & & & & 0.001 \\
\hline 0 & 103 & 61.3 & 133 & 78.7 & \\
\hline 1 & 51 & 30.4 & 24 & 14.2 & \\
\hline 2 & 14 & 8.3 & 12 & 7.1 & \\
\hline
\end{tabular}


Table. 3 Comparison of PD-1/PD-L1 expression pattern between $\mathrm{dMMR}$ and PMMR CRC patients.

\begin{tabular}{|c|c|c|c|c|c|}
\hline \multirow[b]{2}{*}{ Variable } & \multicolumn{2}{|l|}{ dMMR } & \multicolumn{2}{|l|}{ pMMR } & \multirow[b]{2}{*}{$P$} \\
\hline & $\begin{array}{l}\text { No. of Patients } \\
(n=168)\end{array}$ & $\%$ & $\begin{array}{l}\text { No. of Patients } \\
(n=169)\end{array}$ & $\%$ & \\
\hline TIL Numbers (/5 & HPF) & & & & 0.011 \\
\hline Mean & 63.4 & & 22.6 & & \\
\hline SD & 58.6 & & 18.4 & & \\
\hline PD-1 (Total) & & & & & 0.504 \\
\hline Positive & 105 & 62.5 & 99 & 58.6 & \\
\hline Negative & 63 & 37.5 & 70 & 41.4 & \\
\hline PD-1 (Tumor) & & & & & 1.000 \\
\hline Positive & 38 & 22.6 & 38 & 22.5 & \\
\hline Negative & 130 & 77.4 & 131 & 77.5 & \\
\hline PD-1 (TIL) & & & & & 0.039 \\
\hline Positive & 92 & 54.8 & 73 & 43.2 & \\
\hline Negative & 76 & 45.2 & 96 & 56.8 & \\
\hline PD-1 (Stroma) & & & & & 0.718 \\
\hline Positive & 49 & 29.2 & 46 & 27.2 & \\
\hline Negative & 119 & 70.8 & 123 & 72.8 & \\
\hline PD-L1 (Total) & & & & & 0.231 \\
\hline Positive & 93 & 55.4 & 82 & 48.5 & \\
\hline Negative & 75 & 44.6 & 87 & 51.5 & \\
\hline PD-L1 (Tumor) & & & & & 0.790 \\
\hline Positive & 36 & 21.4 & 34 & 20.1 & \\
\hline Negative & 132 & 78.6 & 135 & 79.9 & \\
\hline PD-L1 ((TIL) & & & & & 0.446 \\
\hline Positive & 82 & 48.8 & 90 & 53.3 & \\
\hline Negative & 86 & 51.2 & 79 & 46.7 & \\
\hline PD-L1 (Stroma) & & & & & $<0.001$ \\
\hline Positive & 37 & 22.0 & 74 & 43.8 & \\
\hline Negative & 131 & 78.0 & 95 & 56.2 & \\
\hline
\end{tabular}

A

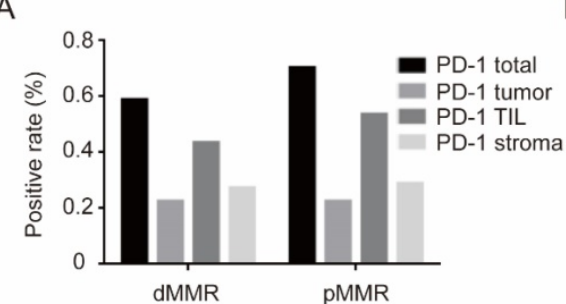

C

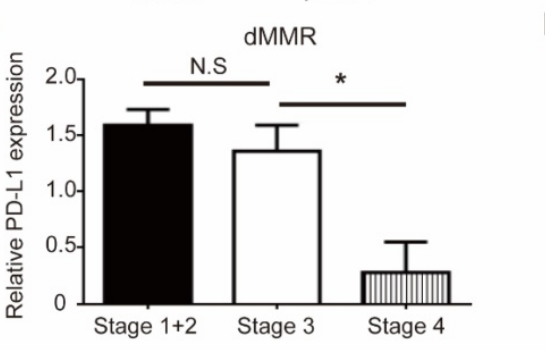

E

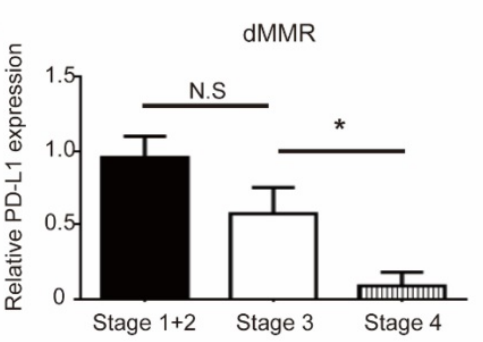

B

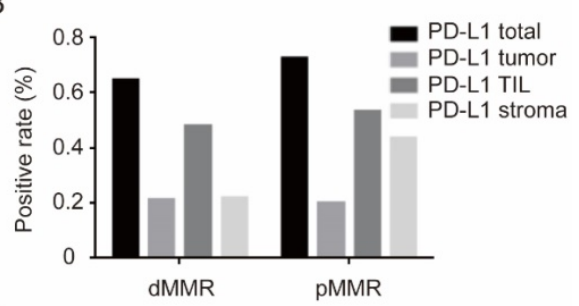

$\mathrm{D}$

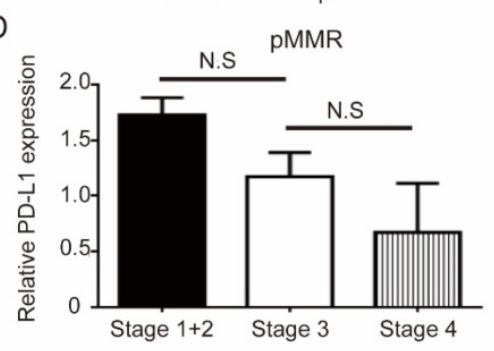

$\mathrm{F}$

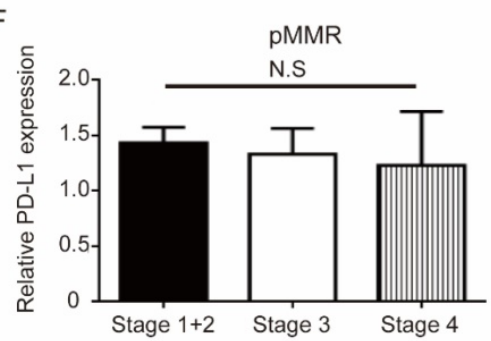

Figure 3. PD-1/PD-L1 expression pattern between dMMR and PMMR CRC patients. (a-b). Distribution of PD-1 (a) or PD-L1 (b) expression for dMMR cohorts $(n=168)$ versus PMMR cohorts $(n=169)$ in CRC patients. (c-d). Group comparison combined the level of TIL PD-L1 and AJCC stages in IMMR (c) or PMMR patients (d). (e-f). Group comparison combined the level of stroma PD-LI (non-TIL and non-tumor) and AJCC stages in dMMR (e) or $\mathrm{PMMR}$ patients $(\mathrm{f})$. $* P<0.05$, N.S, not significant.

\section{Discussion}

The current study comprehensively explored systemic inflammatory responses and PD-1/PD-L1 pathway-related tumor immune microenvironment between $\mathrm{dMMR}$ and pMMR CRC, and evaluated the potential of these features as prognostic factors for CRC. We found that different MSI status was associated with different systemic inflammatory responses and PD-L1/PD-L1 pathway-related TME in CRC. Furthermore, our study suggested PD-L1 expression on TIL was associated with OS and DFS of CRC patients.

A high level of systemic inflammation status and tumor immune cell infiltrates has previously been described with dMMR CRC patients [26-28]. For example, the inflammatory reaction markers such as GPS and NPS is significantly different in MSI vs MSS colorectal tumors[26]. Consistently, we also observed elevated levels of serum CRP, NLR, and GPS (Score 1) in $\mathrm{dMMR}$ group. It is also reported that NLR predicts DFS and OS and is associated with a more aggressive tumor phenotype, and the lymphocytic response to tumor at the invasive margin (IM) is associated with NLR [27]. However, NLR was not associated with prognosis in our study, and we only observed that the circulating serum lymphocytes could predict OS in CRC patients (Table 4), and participants of advanced AJCC stage with relative low level in dMMR cohorts (Figure 2B). The systemic inflammation is generally associated with poor prognosis, emanating from inhibitory immune reaction along tumor progression. Whilst lymphocytes are often representing adaptive immune response controlling tumor. Our findings are concordant with such concept. The difference of DFS did not reach significance between two serum lymphocytes groups might be due to limited period of follow-up information collection. Interestingly, the prognosis of low level of circulating lymphocytes predicts short OS and DFS in dMMR CRC group, but not in pMMR group (Figure 4). This might imply that adaptive immune control of cancer is more prominent in dMMR CRC, of which loss of such control has a significant impact. 
Table. 4 Univariate and Multivariate analyses of prognostic factors for overall survival of CRC patients.

\begin{tabular}{|c|c|c|c|c|c|c|}
\hline \multirow[b]{2}{*}{ Characteristic } & \multicolumn{3}{|c|}{ Univariate analysis } & \multicolumn{3}{|c|}{ Multivariate analysis } \\
\hline & HR & 95\% CI & $P$ & HR & $95 \% \mathrm{CI}$ & $P$ \\
\hline $\begin{array}{l}\text { Regional LNs } \\
\text { metastasis } \geq \mathrm{N} 1\end{array}$ & 3.86 & $(1.65,9.03)$ & 0.002 & 1.18 & $(0.28,4.91)$ & 0.824 \\
\hline Distant metastasis & 15.09 & $(6.53,34.86)$ & $<0.001$ & 5.23 & $(1.54,17.83)$ & 0.008 \\
\hline AJCC stage $\geq 3$ & 14.93 & $(4.42,50.48)$ & $<0.001$ & 7.35 & $(1.01,53.69)$ & 0.049 \\
\hline Vascular invasion & 6.55 & $(2.54,16.86)$ & $<0.001$ & 4.98 & $(1.63,15.24)$ & 0.005 \\
\hline Chemotherapy & 4.06 & $(1.48,11.11)$ & 0.006 & 1.68 & $(0.51,5.55)$ & 0.394 \\
\hline Radiotherapy & 6.62 & $(2.69,16.30)$ & $<0.001$ & 4.40 & $(1.49,12.93)$ & 0.006 \\
\hline $\begin{array}{l}\text { CA19-9 }(\geq 30 \\
\text { units } / \mathrm{ml})\end{array}$ & 2.49 & $(1.05,5.95)$ & 0.040 & 2.02 & $(0.69,5.91)$ & 0.198 \\
\hline $\begin{array}{l}\text { Lymphocyte } \\
\left(\geq 1.12 \times 10^{9} / 1\right)\end{array}$ & 4.45 & $(1.32,15.06)$ & 0.016 & 4.82 & $(1.12,20.69)$ & 0.035 \\
\hline TIL Numbers $(>40)$ & 0.44 & $(0.19,1.03)$ & 0.058 & - & - & - \\
\hline $\begin{array}{l}\text { PD-L1 expression } \\
\text { (Total) }\end{array}$ & 0.40 & $(0.17,0.93)$ & 0.033 & 0.46 & $(0.12,1.77)$ & 0.257 \\
\hline $\begin{array}{l}\text { PD-L1 expression } \\
\text { (TIL) }\end{array}$ & 0.69 & $(0.49,0.96)$ & 0.026 & 0.66 & $(0.21,2.05)$ & 0.469 \\
\hline
\end{tabular}

It is not unexpected to find the significant difference of PD-L1/PD-L1 expression patterns in dMMR and pMMR CRC patients. First, our study clearly showed that, despite similar distribution of
A

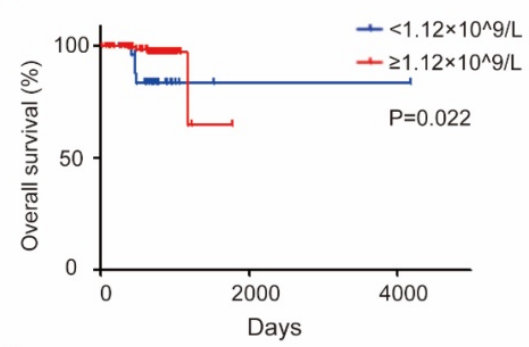

C

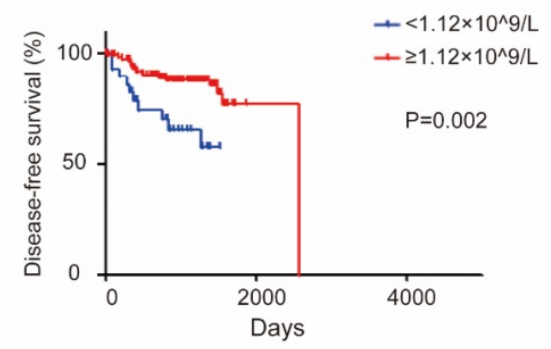

E

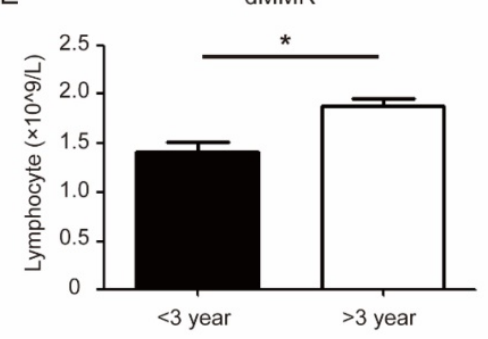

B

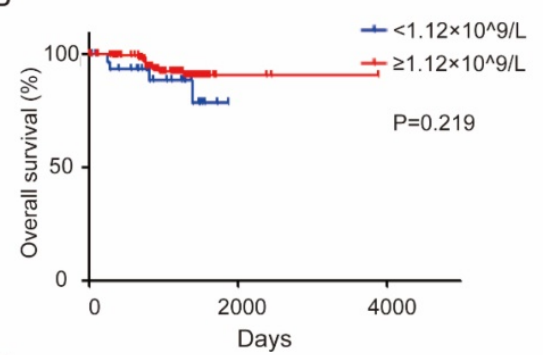

D

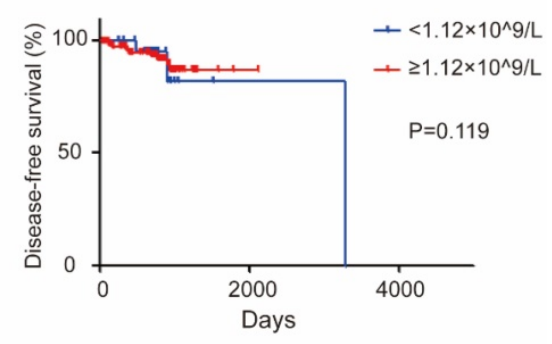

$\mathrm{F}$

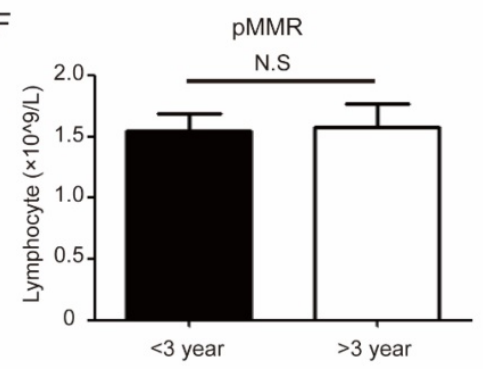

Figure 4. The different association of circulating lymphocyte level and prognosis between $d M M R$ and PMMR CRC patients. (a-b). Kaplan-Meier survival curves comparing the overall survival of the level of circulating lymphocytes in $\operatorname{dMMR}$ (a) or PMMR CRC patients (b). (c-d) Kaplan-Meier survival curves comparing the disease-free survival of the level of circulating lymphocytes in DMMR (c) or PMMR CRC patients (d). Group comparison (Disease-free survival: $<3$ year, $n=92$ vs $>3$ year, $n=76$ ) combined the level of lymphocytes and different disease-free survival in dMMR (e). Group comparison (Disease-free survival: < 3 year, $n=154$ vs $>3$ year, $n=15$ ) combined the level of lymphocytes and different disease-free survival in pMMR patients (f). $* P<0.05$, N.S, not significant.
PD-1 and PD-L1 in different area, PD-L1 expression on TIL and stroma (non-TIL and non-tumor) was significantly different between $\mathrm{dMMR}$ and pMMR CRC (Figure 3A-B). This is consistent with previous report that PD-L1 expression was largely on TILs but not tumor cells in dMMR CRC tumors [29]. Second, our study indicated that a potential inverse correlation between TIL PD-L1 or stroma PD-L1 and AJCC stage in dMMR patients (Figure 3C-F). We speculate the conspicuous drop of PD-L1 in AJCC stage 4 patients with concurrent decreased lymphocyte level may indicate a undermined antitumor immunity in late stage patients, and PD-1/PD-L1 pathway may weigh less in tumor immunity these patients. One recent report found that TIL PD-1 is an independent prognostic factor for OS and DFS of pMMR CRC patients, but not dMMR [30]. The authors suggested that TIL-PD1 might be associated with the regulatory and immune-suppressive $\mathrm{T}$ cells within colon microenvironment during an enhanced antitumor immune response. However, tumor PD-L1 expression was found inversely associated with FOXP3+ cell density in CRCs [31], which further confound the implications of such findings. By contrast, we only found higher expressions of total PD-L1 and TIL PD-L1 expression correlates with better OS and DFS of CRC patients but none serves as an independent prognostic factor for prognosis. Several potential reasons might explain the different result of our study. Firstly, we noticed that the studies used antibodies from different vendors, and the results were obtained from both TCGA database and tissue microarray [31, 32]. The technical variations in tissue staining could introduce some difference. Secondly, our data are obtained from cancer registries from a single center, which may introduce some degree of selection bias. Thirdly, our results of distribution analysis also displayed that, compared to pMMR cohorts, expression of total PD-L1decreased sharply in the AJCC stage 4, which implied that the importance of PD-1/PD-L1 in anti-tumor immunity might vary in different AJCC stages. 
Table. 5 Univariate and Multivariate analyses of prognostic factors for disease-free survival of CRC patients.

\begin{tabular}{|c|c|c|c|c|c|c|}
\hline \multirow[b]{2}{*}{ Characteristic } & \multicolumn{3}{|c|}{ Univariate analysis } & \multicolumn{3}{|c|}{ Multivariate analysis } \\
\hline & HR & 95\% CI & $P$ & HR & $95 \% \mathrm{CI}$ & $P$ \\
\hline $\begin{array}{l}\text { Regional LNs } \\
\text { metastasis } \geq \mathrm{N} 1\end{array}$ & 3.67 & $(2.09,6.47)$ & $<0.001$ & 1.96 & $(0.58,6.61)$ & 0.278 \\
\hline Distant metastasis & 8.25 & $(4.36,15.60)$ & $<0.001$ & 4.39 & $(1.58,12.21)$ & 0.005 \\
\hline AJCC stage $\geq 3$ & 5.63 & $(3.07,10.34)$ & $<0.001$ & 1.89 & $(0.47,7.57)$ & 0.368 \\
\hline Vascular invasion & 2.70 & $(1.34,5.44)$ & 0.005 & 1.77 & $(0.83,3.76)$ & 0.139 \\
\hline $\begin{array}{l}\text { Number of retrieved } \\
\text { LNs }<12\end{array}$ & 2.43 & $(1.39,4.25)$ & 0.002 & 2.01 & $(1.00,4.03)$ & 0.049 \\
\hline Chemotherapy & 3.27 & $(1.73,6.19)$ & $<0.001$ & 1.23 & $(0.58,2.59)$ & 0.593 \\
\hline Radiotherapy & 8.56 & $(4.58,15.99)$ & $<0.001$ & 6.80 & $(2.86,16.17)$ & $<0.001$ \\
\hline CEA $(\geq 5 \mu \mathrm{g} / \mathrm{ml})$ & 1.38 & $(1.03,1.85)$ & 0.029 & 0.82 & $(0.55,1.22)$ & 0.329 \\
\hline $\begin{array}{l}\text { CA19-9 }(\geq 30 \\
\text { units } / \mathrm{ml})\end{array}$ & 2.06 & $(1.12,3.79)$ & 0.020 & 1.35 & $(0.96,1.89)$ & 0.081 \\
\hline $\begin{array}{l}\text { Lymphocyte } \\
\left(\geq 1.12 \times 10^{9} / 1\right)\end{array}$ & 1.32 & $(1.07,1.61)$ & 0.008 & 0.87 & $(0.66,1.15)$ & 0.320 \\
\hline TIL Numbers (>40) & 0.86 & $(0.31,2.28)$ & 0.346 & - & - & - \\
\hline $\begin{array}{l}\text { PD-L1 expression } \\
\text { (Total) }\end{array}$ & 0.40 & $(0.17,0.93)$ & 0.033 & 1.09 & $(0.84,1.41)$ & 0.513 \\
\hline $\begin{array}{l}\text { PD-L1 expression } \\
\text { (TIL) }\end{array}$ & 0.51 & $(0.28,0.92)$ & 0.025 & 0.98 & $(0.75,1.28)$ & 0.863 \\
\hline
\end{tabular}

One important finding of our study is that the PD-L1 expression at different location has different impact on survival in CRC patients. PD-L1 is usually expressed by $\mathrm{T}$ and $\mathrm{B}$ cells, macrophages, dendritic cells (DC) and other normal cells [33]. PD-L1 is also expressed in many cancers, and is strongly associated

A
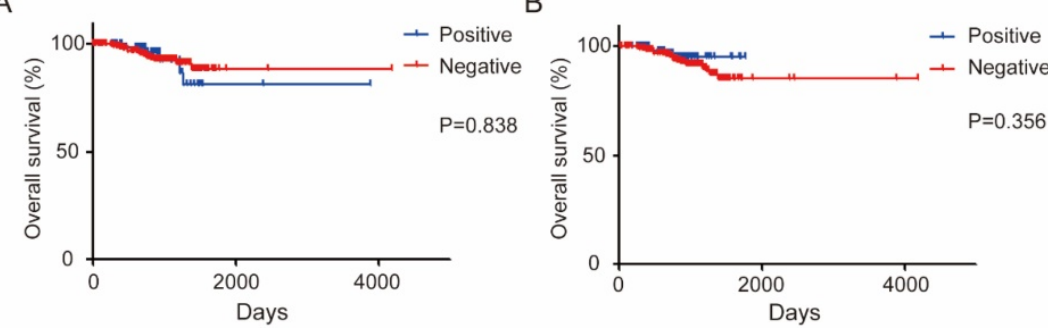

C
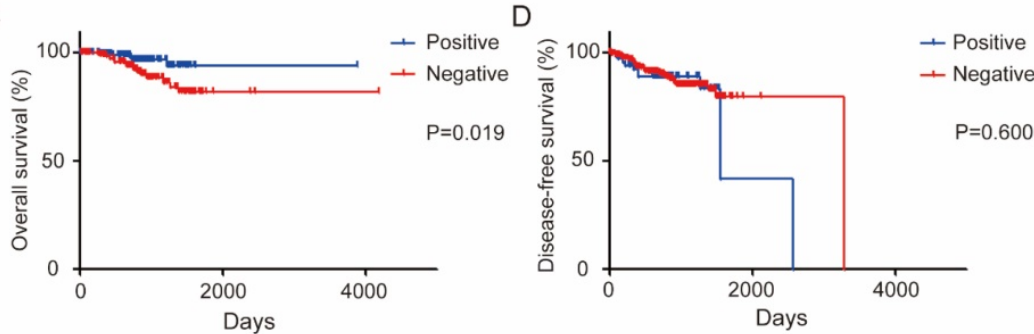

$E$

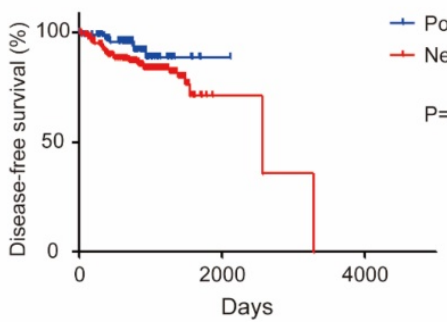

$\mathrm{F}$

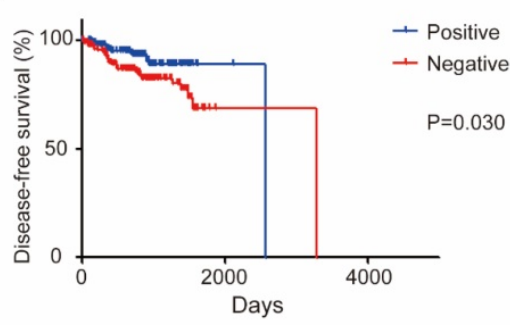

Figure 5. Localized PD-L1 expression is associated with CRC patient survival. (a-c). Kaplan-Meier survival curves comparing the overall survival of the level of tumor PD-L1 (a), stroma PD-LI (b), or TIL PD-L1 (c) expression in CRC patients. (d-f). Kaplan-Meier survival curves comparing the disease-free survival of the level of tumor PD-LI (d), stroma PD-LI (e), or TIL PD-LI (f) expression in CRC patients. with poor prognosis[34-37]. Furthermore, the expression of PD-L1 in tumor cells (TCs) has been identified as a predictive factor for tumor response to anti-PD-1 or anti-PD-L1 immunotherapy in various malignancies[13, 38-40]. For CRC, it was reported that patients with higher expression of PD-L1 had a poorer prognosis in colorectal serrated adenocarcinoma[41], in which PD-L1 could be one strategy for tumors to evade from immune attack. On the other hand, PD-L1 induction in colorectal cancer cells are blunted comparing to melanoma cells, while TILs and myeloid cells seemed more amenable to IFN $\gamma$-induced PD-L1 expression in colorectal tumors [29]. Thus, PD-L1 expression on TILs maybe a reflection of effector T cell activity. Consistently, several recent studies suggested PD-L1 expression is paradoxically associated with favorable survival in CRC, especially the TIL PD-L1[21, 30]. In a recent report, careful examination of localized PD1 and PD-L1 in CRC tumors suggested that high PD-1-positve TILs is associated with improved survival only when tumors have low level PD-L1 expression, and both high PD-1 positive TILs and high PD-L1 expression predicts worse recurrence free survival [42]. In our study, we found that total PD-1 was not a prognostic marker, but the total PD-L1 was a good prognostic marker in our cohorts, and high TIL PD-L1 expression significantly predicts a favorable prognosis in CRC patients (Figure 5C). Our result further supported the idea that PD-L1 on TIL may likely reflect the intratumor effector $\mathrm{T}$ cell activation, a major mechanism by which adaptive immune system controlling tumor. Lastly, our outcomes manifested that different area of PD-L1 with different impact on DFS in CRC and high TIL PD-L1 expression was significantly associated with long DFS in CRC $(\mathrm{P}=0.030)$ (Figure 5). Nevertheless, as the potential limitation of statistic power, the multivariate analysis results indicated total PD-PD-L1 and TIL PD-L1 were not the independent prognostic factor for survival. Hence, our study first demonstrated that, unlike previous studies, PD-L1 expression on specific area such like TIL PD-L1 might be a good predictor for prognosis in CRC.

To date there is no reliable prediction markers for patient response to PD-1 blockade. Although CD8 T cell infiltrates and intra-tumor PD-L1 expression was considered one of the 
most prominent features of responders, more in-depth analyses suggested localized PD-L1 expression on TILs and PD-L1/PD-1 proximity were more accurate in predicting melanoma patient response [38, 43]. TME in dMMR CRC patients was featured with abundant TIL infiltrates and elevated levels of multiple inhibitory molecules such as PD-1, PD-L1, LAG3, IDO-1, etc. which serve as a good target for PD-1 blockade [29]. Indeed, clinical trial result confirmed the hypersensitivity of these patients to anti-PD1 treatment. However, the response rate was still limited (4 out of 10 patients), and the histology data about intra-tumor PD-L1 expression was not clear [14]. Therefore, the value of intratumor PD-L1 expression in predicting response to PD-1 blockade warrant further investigation from clinical trials on more dMMR CRC patients. In addition, the systemic immune status pre-treatment may predict anti-PD1 response, as suggested by recent reports that high levels of circulating MDSCs or Ki67+PD1+CD8 T cells is associated with unfavorable or favorable response to immunotherapy. Whether such association also exist in CRC patients remains to be investigated.

Despite our study's trustworthy strengths, including a large sample size, reliable follow-up information, primary tumor tissue slides, and full-scale molecular characterization, there are several limitations. Firstly, the main limitation is no consistent and accurate criteria for PD-L1 and TIL positive diagnosis. Although we diagnose these markers strictly based on previous established criteria and try our best to minimize diagnostic related error, we cannot guarantee absolute accurate diagnosis [23, $30,44]$. Secondly, our sample data are not derived from an RCT, and the data are limited by non-uniform of patient treatment and follow-up for events, and we did not analyze detailed treatment patterns or all classical features that have previously been noted as potential prognostic factors. Thirdly, we were not able to include any patients with PD-1/PD-L1 blockade therapy, leaving the prediction value of intratumor PD-L1 expression uncertain at this moment.

Overall, our study indicated a distinct TME characteristics is associated with dMMR CRC at specific disease stages, and that the PD-L1 expression on TIL may have a positive impact on survival of CRC patient, and worth future study for its prediction value for choosing right patients for PD-1/PD-L1 blockade therapy.

\section{Supplementary Material}

Supplementary figures and tables.

http://www.jcancer.org/v10p1745s1.pdf

\section{Acknowledgements}

We thank Dr. Ruiyan Wu for statistical advising and review of the manuscript.

\section{Funding}

This work was supported in part by funding from: Natural Science Foundation of Guangdong (2015A030313010 to L.X.), Science and Technology Program of Guangzhou, China (1563000305 to L.X.) and National Natural Science Foundation of China (81272641 and 81572409 to L.X., 81472578 to X.X.).

\section{Ethics committee approval and patient consent}

This study was approved by Ethics Committee and Institutional Review Board of SYSUCC, and written informed consent was obtained from each patient included in the study.

\section{Competing Interests}

The authors have declared that no competing interest exists.

\section{References}

1. Schumacher TN, Schreiber RD. Neoantigens in cancer immunotherapy. Science. 2015; 348: 69-74.

2. McGranahan N, Furness AJ, Rosenthal R, Ramskov S, Lyngaa R, Saini SK, et al. Clonal neoantigens elicit $\mathrm{T}$ cell immunoreactivity and sensitivity to immune checkpoint blockade. Science. 2016; 351: 1463-9.

3. Stronen E, Toebes M, Kelderman S, van Buuren MM, Yang W, van Rooij N, et al. Targeting of cancer neoantigens with donor-derived $\mathrm{T}$ cell receptor repertoires. Science. 2016; 352: 1337-41.

4. Manzanillo P, Eidenschenk C, Ouyang W. Deciphering the crosstalk among IL-1 and IL-10 family cytokines in intestinal immunity. Trends in immunology. 2015; 36: 471-8.

5. Flavell RA, Sanjabi S, Wrzesinski SH, Licona-Limon P. The polarization of immune cells in the tumour environment by TGFbeta. Nature reviews Immunology. 2010; 10: 554-67.

6. Freeman GJ, Long AJ, Iwai Y, Bourque K, Chernova T, Nishimura $\mathrm{H}$, et al. Engagement of the PD-1 immunoinhibitory receptor by a novel B7 family member leads to negative regulation of lymphocyte activation. The Journal of experimental medicine. 2000; 192: 1027-34.

7. Robert C, Ribas A, Wolchok JD, Hodi FS, Hamid O, Kefford R, et al. Anti-programmed-death-receptor-1 treatment with pembrolizumab in ipilimumab-refractory advanced melanoma: a randomised dose-comparison cohort of a phase 1 trial. Lancet. 2014; 384: 1109-17.

8. McDermott DF, Sosman JA, Sznol M, Massard C, Gordon MS, Hamid O, et al. Atezolizumab, an Anti-Programmed Death-Ligand 1 Antibody, in Metastatic Renal Cell Carcinoma: Long-Term Safety, Clinical Activity, and Immune Correlates From a Phase Ia Study. Journal of clinical oncology : official journal of the American Society of Clinical Oncology. 2016; 34: 833-42.

9. Samadder NJ, Vierkant RA, Tillmans LS, Wang AH, Weisenberger DJ, Laird PW, et al. Associations between colorectal cancer molecular markers and pathways with clinicopathologic features in older women. Gastroenterology. 2013; 145: 348-56 e1-2.

10. Rosenberg JE, Hoffman-Censits J, Powles T, van der Heijden MS, Balar AV, Necchi A, et al. Atezolizumab in patients with locally advanced and metastatic urothelial carcinoma who have progressed following treatment with platinum-based chemotherapy: a single-arm, multicentre, phase 2 trial. Lancet. 2016; 387: 1909-20.

11. Fehrenbacher L, Spira A, Ballinger M, Kowanetz M, Vansteenkiste J, Mazieres $\mathrm{J}$, et al. Atezolizumab versus docetaxel for patients with previously treated non-small-cell lung cancer (POPLAR): a multicentre, open-label, phase 2 randomised controlled trial. Lancet. 2016; 387: 1837-46.

12. Herbst RS, Baas P, Kim DW, Felip E, Perez-Gracia JL, Han JY, et al. Pembrolizumab versus docetaxel for previously treated, PD-L1-positive, advanced non-small-cell lung cancer (KEYNOTE-010): a randomised controlled trial. Lancet. 2016; 387: 1540-50.

13. Topalian SL, Hodi FS, Brahmer JR, Gettinger SN, Smith DC, McDermott DF, et al. Safety, activity, and immune correlates of anti-PD-1 antibody in cancer. The New England journal of medicine. 2012; 366: 2443-54. 
14. Le DT, Uram JN, Wang H, Bartlett BR, Kemberling H, Eyring AD, et al. PD-1 Blockade in Tumors with Mismatch-Repair Deficiency. The New England journal of medicine. 2015; 372: 2509-20.

15. Fleuren GJ, Gorter A, Kuppen PJ, Litvinov S, Warnaar SO. Tumor heterogeneity and immunotherapy of cancer. Immunological reviews. 1995; 145: 91-122.

16. Haradhvala NI, Polak P, Stojanov P, Covington KR, Shinbrot E, Hess JM, et al. Mutational Strand Asymmetries in Cancer Genomes Reveal Mechanisms of DNA Damage and Repair. Cell. 2016; 164: 538-49.

17. Zou W, Wolchok JD, Chen L. PD-L1 (B7-H1) and PD-1 pathway blockade for cancer therapy: Mechanisms, response biomarkers, and combinations. Science translational medicine. 2016; 8: 328rv4.

18. Lee SY, Chung H, Devaraj B, Iwaizumi M, Han HS, Hwang DY, et al. Microsatellite alterations at selected tetranucleotide repeats are associated with morphologies of colorectal neoplasias. Gastroenterology. 2010; 139: 1519-25.

19. Kim JC, Koo KH, Kim HC, Kim JS, Kang GH. Geno- and pheno-typic characterization in ten patients with double-primary gastric and colorectal adenocarcinomas. International journal of colorectal disease. 2004; 19: 561-8.

20. Gill S, Lindor NM, Burgart LJ, Smalley R, Leontovich O, French AJ, et al. Isolated loss of PMS2 expression in colorectal cancers: frequency, patient age, and familial aggregation. Clinical cancer research : an official journal of the American Association for Cancer Research. 2005; 11: 6466-71.

21. Droeser RA, Hirt C, Viehl CT, Frey DM, Nebiker C, Huber X, et al. Clinical impact of programmed cell death ligand 1 expression in colorectal cancer. European journal of cancer. 2013; 49: 2233-42.

22. Taube JM, Klein A, Brahmer JR, Xu H, Pan X, Kim JH, et al. Association of PD-1, PD-1 ligands, and other features of the tumor immune microenvironment with response to anti-PD-1 therapy. Clinical cancer research : an official journal of the American Association for Cancer Research. 2014; 20: 5064-74.

23. Yang CY, Lin MW, Chang YL, Wu CT, Yang PC. Programmed cell death-ligand 1 expression in surgically resected stage I pulmonary adenocarcinoma and its correlation with driver mutations and clinical outcomes. European journal of cancer. 2014; 50: 1361-9.

24. Rozek LS, Schmit SL, Greenson JK, Tomsho LP, Rennert HS, Rennert G, et al. Tumor-Infiltrating Lymphocytes, Crohn's-Like Lymphoid Reaction, and Survival From Colorectal Cancer. Journal of the National Cancer Institute. 2016; 108.

25. Ishizuka M, Nagata H, Takagi K, Iwasaki Y, Shibuya N, Kubota K. Clinical Significance of the C-Reactive Protein to Albumin Ratio for Survival After Surgery for Colorectal Cancer. Annals of surgical oncology. 2016; 23: 900-7.

26. De Smedt L, Lemahieu J, Palmans S, Govaere O, Tousseyn T, Van Cutsem E, et al. Microsatellite instable vs stable colon carcinomas: analysis of tumour heterogeneity, inflammation and angiogenesis. British journal of cancer. 2015; 113: 500-9.

27. Pine JK, Morris E, Hutchins GG, West NP, Jayne DG, Quirke P, et al. Systemic neutrophil-to-lymphocyte ratio in colorectal cancer: the relationship to patient survival, tumour biology and local lymphocytic response to tumour. British journal of cancer. 2015; 113: 204-11.

28. Park JH, Powell AG, Roxburgh CS, Horgan PG, McMillan DC, Edwards J. Mismatch repair status in patients with primary operable colorectal cancer: associations with the local and systemic tumour environment. British journal of cancer. 2016; 114: 562-70.

29. Llosa NJ, Cruise M, Tam A, Wicks EC, Hechenbleikner EM, Taube JM, et al. The vigorous immune microenvironment of microsatellite instable colon cancer is balanced by multiple counter-inhibitory checkpoints. Cancer discovery. 2015; 5: 43-51.

30. Li Y, Liang L, Dai W, Cai G, Xu Y, Li X, et al. Prognostic impact of programed cell death-1 (PD-1) and PD-ligand 1 (PD-L1) expression in cancer cells and tumor infiltrating lymphocytes in colorectal cancer. Molecular cancer. 2016; 15:

31. Masugi Y, Nishihara R, Yang J, Mima K, da Silva A, Shi Y, et al. Tumour CD274 (PD-L1) expression and T cells in colorectal cancer. Gut. 2016.

32. Zhang H, Liu T, Zhang Z, Payne SH, Zhang B, McDermott JE, et al. Integrated Proteogenomic Characterization of Human High-Grade Serous Ovarian Cancer. Cell. 2016; 166: 755-65.

33. Keir ME, Liang SC, Guleria I, Latchman YE, Qipo A, Albacker LA, et al. Tissue expression of PD-L1 mediates peripheral $\mathrm{T}$ cell tolerance. The Journal of experimental medicine. 2006; 203: 883-95.

34. Thompson RH, Kuntz SM, Leibovich BC, Dong H, Lohse CM, Webster WS, et al. Tumor B7-H1 is associated with poor prognosis in renal cell carcinoma patients with long-term follow-up. Cancer research. 2006; 66: 3381-5.

35. Wu K, Kryczek I, Chen L, Zou W, Welling TH. Kupffer cell suppression of CD8+ $\mathrm{T}$ cells in human hepatocellular carcinoma is mediated by B7-H1/programmed death-1 interactions. Cancer research. 2009; 69: 8067-75.

36. Rossille D, Gressier M, Damotte D, Maucort-Boulch D, Pangault C, Semana G, et al. High level of soluble programmed cell death ligand 1 in blood impacts overall survival in aggressive diffuse large B-Cell lymphoma: results from a French multicenter clinical trial. Leukemia. 2014; 28: 2367-75.

37. Kiyasu J, Miyoshi H, Hirata A, Arakawa F, Ichikawa A, Niino D, et al. Expression of programmed cell death ligand 1 is associated with poor overall survival in patients with diffuse large B-cell lymphoma. Blood. 2015; 126: 2193-201.
38. Herbst RS, Soria JC, Kowanetz M, Fine GD, Hamid O, Gordon MS, et al. Predictive correlates of response to the anti-PD-L1 antibody MPDL3280A in cancer patients. Nature. 2014; 515: 563-7.

39. Awad MM, Hammerman PS. Durable Responses With PD-1 Inhibition in Lung and Kidney Cancer and the Ongoing Search for Predictive Biomarkers. Journal of clinical oncology : official journal of the American Society of Clinical Oncology. 2015; 33: 1993-4.

40. Powles T, Eder JP, Fine GD, Braiteh FS, Loriot Y, Cruz C, et al. MPDL3280A (anti-PD-L1) treatment leads to clinical activity in metastatic bladder cancer. Nature. 2014; 515: 558-62.

41. Zhu H, Qin H, Huang Z, Li S, Zhu X, He J, et al. Clinical significance of programmed death ligand-1 (PD-L1) in colorectal serrated adenocarcinoma. International journal of clinical and experimental pathology. 2015; 8: 9351-9.

42. Lee LH, Cavalcanti MS, Segal NH, Hechtman JF, Weiser MR, Smith JJ, et al. Patterns and prognostic relevance of PD-1 and PD-L1 expression in colorectal carcinoma. Mod Pathol. 2016; 29: 1433-42.

43. Tumeh PC, Harview CL, Yearley JH, Shintaku IP, Taylor EJ, Robert L, et al. PD-1 blockade induces responses by inhibiting adaptive immune resistance. Nature. 2014; 515: 568-71.

44. Ogino S, Nosho K, Irahara N, Meyerhardt JA, Baba Y, Shima K, et al. Lymphocytic reaction to colorectal cancer is associated with longer survival, independent of lymph node count, microsatellite instability, and CpG island methylator phenotype. Clinical cancer research : an official journal of the American Association for Cancer Research. 2009; 15: 6412-20. 